Pacific Journal of Mathematics

CONCERNING HYPERSPACES OF CERTAIN PLANO
CONTINUA AND STRONG REGULARITY OF WHITEY MAPS 


\title{
CONCERNING HYPERSPACES OF CERTAIN PEANO CONTINUA AND STRONG REGULARITY OF WHITNEY MAPS
}

\author{
Hisao Kato
}

Let $X$ be a Peano continuum and let $\mathfrak{E}=2^{X}$ (resp., $C(X)$ ) be the space of all nonempty closed subsets (resp., subcontinua) of $X$ with Hausdorff metric. If $\mathfrak{E}=C(x)$, assume that $X$ contains no free arc. Then the following are shown.

(1) If $\omega$ is an admissible Whitney map for $\mathfrak{g}$, then

$$
\omega \mid \omega^{-1}((0, \omega(X))): \omega^{-1}((0, \omega(X))) \rightarrow(0, \omega(X))
$$

is a trivial bundle map with Hilbert cube fibers.

(2) If $X$ is the Hilbert cube $Q$, then there is a strongly admissible Whitney map $\omega$ for $\mathfrak{g}$ such that $\omega / \omega^{-1}([0, \omega(X))) \rightarrow[0, \omega(X))$ is a trivial bundle map with Hilbert cube fibers.

(3) If $X$ is the $n$-sphere $S^{n}(n=1,2, \ldots$,$) , then there is a Whitney$ map $\omega$ for $2^{X}$ such that for some $t_{0} \in(0, \omega(X)), \omega \mid \omega^{-1}\left(\left(0, t_{0}\right]\right)$ : $\omega^{-1}\left(\left(0, t_{0}\right]\right) \rightarrow\left(0, t_{0}\right]$ is a trivial bundle map with $X \times Q$ fibers. If $X$ is the $n$-sphere $S^{n}(n=2,3, \ldots$,$) , there is a Whitney map \omega$ for $C(X)$ such that for some $t_{0} \in(0, \omega(X)), \omega \mid \omega^{-1}\left(\left(0, t_{0}\right]\right)$ is a trivial bundle map with $X \times Q$ fibers.

1. Introduction. Throughout this paper, the word compactum means a compact metric space. A connected compactum is a continuum. A Peano continuum is a locally connected continuum. If $x$ and $y$ are points of a metric space, $d(x, y)$ denotes the metric from $x$ to $y$. For any subsets $A$ and $B$ of a metric space, let $d(A, B)=\inf \{d(a, b) \mid a \in A, b \in B\}$. Also, let $d_{H}(A, B)=\max \left\{\sup _{a \in A} d(a, B), \sup _{b \in B} d(b, A)\right\} . d_{H}$ is called the Hausdorff metric. The hyperspaces of a continuum $X$ are the spaces $2^{X}=\{A \subset X \mid A$ is nonempty and compact $\}$ and $C(X)=\left\{A \in 2^{X} \mid A\right.$ is connected $\}$ which are metrized with the Hausdorff metric $d_{H}$. Let $F_{1}(X)$ $=\{\{x\} \mid x \in X\}$. A Whitney map for a hyperspace $\mathfrak{S}$ of a continuum $X$ is a continuous function $\omega: \mathfrak{S} \rightarrow[0, \omega(X)]$ such that $\omega(\{x\})=0$ for each $\{x\} \in F_{1}(X)$, and if $A, B \in \mathfrak{S}_{\mathfrak{g}}$ and $A \subsetneq B$, then $\omega(A)<\omega(B)$ (see [8, 13]). The notion of Whitney map is a convenient and important tool for hyperspace theory. If $\omega$ is a Whitney map for $\mathfrak{g}=2^{X}$ or $C(X)$ and $0 \leq t<\omega(X)$, then $\omega^{-1}(t)$ is called a Whitney level. Note that Whitney levels are coverings of $X$ which, as $t$ gets close to zero, converge to $\omega^{-1}(0)=F_{1}(X) \cong X$. It is of interest to obtain information about the 
structure of Whitney levels and to determine those properties which are preserved by the convergence of positive Whitney levels to the zero level. Throughout this paper, by $Q$ we mean the Hilbert cube $\prod_{i=1}^{\infty}[-1,1]$. In [4], Curtis and Schori proved that for any Peano continuum $X, 2^{X}$ is a Hilbert cube and if $X$ contains no free arc, $C(X)$ is also a Hilbert cube. Recently, Goodykoontz and Nadler introduced a very important notion "admissible Whitney map" to study the structure of certain Whitney maps. Let $\mathfrak{S}=2^{X}$ or $C(X)$. A Whitney map $\omega$ for $\mathfrak{S}$ is an admissible Whitney map [7] if there is a homotopy $h: \mathfrak{S} \times[0,1] \rightarrow \mathfrak{S}$ satisfying the following two conditions;

(1) for all $A \in \mathfrak{S}_{\mathfrak{g}}, h(A, 1)=A, h(A, 0) \in F_{1}(X)$, and

(2) if $\omega(h(A, t))>0$ for some $A \in \mathfrak{S}, t \in(0,1]$, then $\omega(h(A, s))<$ $\omega(h(A, t))$ whenever $0 \leq s<t \leq 1$.

Such a homotopy $h: \mathfrak{S} \times[0,1] \rightarrow \mathfrak{S}$ is called an $\omega$-admissible deformation for $\mathfrak{S}$. In [7], it is shown that if $X$ is either a compact starshaped subset of a Banach space or a dendrite, then there exist admissible Whitney maps for $2^{X}$ and $C(X)$. Also, if $X$ is any smooth dendroid, then every Whitney map for $C(X)$ is admissible. By using this notion, Goodykoontz and Nadler proved the following very interesting results.

(1.1) THEOREM ([7]). Let $X$ be a Peano continuum and let $\omega$ be an admissible Whitney map for $\mathfrak{S}=2^{X}$ or $C(X)$. If $\mathfrak{S}=C(X)$, assume that $X$ contains no free arc. Then for any $t \in(0, \omega(X)), \omega^{-1}(t), \omega^{-1}([0, t])$ and $\omega^{-1}([t, \omega(X)])$ are Hilbert cubes.

(1.2) THEOREM ([7]). If $\omega$ is an admissible Whitney map for $\mathfrak{S}=2^{X}$ or $C(X)$, then $\omega$ is an open map with FAR fibers.

The purpose of this paper is to give more precise information by using infinite-dimensional topology. The ideas and techniques in this paper essentially depend on [7]. Our main results are the following: Let $X$ be a Peano continuum and let $\mathfrak{S}=2^{X}$ or $C(X)$. If $\mathfrak{S}=C(X)$, assume that $X$ contains no free arc.

(1) If $\omega$ is an admissible Whitney map for $\mathfrak{g}$, then $\omega \mid \omega^{-1}((0, \omega(X)))$ : $\omega^{-1}((0, \omega(X))) \rightarrow(0, \omega(X))$ is a trivial bundle map with Hilbert cube fibers.

(2) If $X$ is the Hilbert cube $Q$, then there is a strongly admissible Whitney map $\omega$ for $\mathfrak{S}$ such that $\omega \mid \omega^{-1}([0, \omega(X))): \omega^{-1}([0, \omega(X))) \rightarrow$ $[0, \omega(X))$ is a trivial bundle map with Hilbert cube fibers. 
(3) If $X$ is the $n$-sphere $S^{n}(n=1,2, \ldots$,$) , then there is a Whitney$ map $\omega$ for $2^{X}$ such that for some $t_{0} \in(0, \omega(X))$, $\omega \mid \omega^{-1}\left(\left(0, t_{0}\right]\right)$ : $\omega^{-1}\left(\left(0, t_{0}\right]\right) \rightarrow\left(0, t_{0}\right]$ is a trivial bundle map with $X \times Q$ fibers. If $X$ is the $n$-sphere $S^{n}(n=2,3, \ldots)$, there is a Whitney map $\omega$ for $C(X)$ such that for some $t_{0} \in(0, \omega(X)), \omega \mid \omega^{-1}\left(\left(0, t_{0}\right]\right): \omega^{-1}\left(\left(0, t_{0}\right]\right) \rightarrow\left(0, t_{0}\right]$ is also a trivial bundle map with $X \times Q$ fibers.

The author wishes to thank the referee for his kind remarks.

2. Strongly regular mappings and Whitney maps. In this section, we show that if $X$ is a Peano continuum and $\omega$ is an admissible Whitney map for $\mathfrak{S}=2^{X}$ or $C(X)$, then the restriction $\omega \mid \omega^{-1}((0, \omega(X)])$ : $\omega^{-1}((0, \omega(X)]) \rightarrow(0, \omega(X)]$ of $\omega$ to $\omega^{-1}((0, \omega(X)])$ is a strongly regular mapping with AR fibers. Also, we introduce the notion "strongly admissible Whitney map" and prove that if $\omega: \mathfrak{G} \rightarrow[0, \omega(X)]$ is a strongly admissible Whitney map, then $\omega$ is a strongly regular mapping with AR fibers.

(2.1) Definition (cf. [7, (2.1)]). Let $X$ be a continuum and let $\mathfrak{S}$ denote either one of the hyperspaces $2^{X}$ or $C(X)$. A Whitney map $\omega$ for $\mathfrak{g}$ is called a strongly admissible Whitney map for $\mathfrak{S}$ provided that there is a homotopy $h: \mathfrak{S} \times[0,1] \rightarrow \mathfrak{S}$ satisfying the following conditions;

(1) $h(\{x\}, s)=\{x\}$ for each $\{x\} \in F_{1}(X)$ and $0 \leq s \leq 1$,

(2) $h(A, 1)=A, h(A, 0) \in F_{1}(X)$ for each $A \in \mathscr{H}$ and

(3) if $\omega(h(A, t))>0$ for some $A \in \mathscr{S}_{\mathcal{B}}$ and $t \in(0,1]$, then $\omega(h(A, s))$ $<\omega(h(A, t))$ for each $0 \leq s<t \leq 1$.

By definitions, every strongly admissible Whitney map is an admissible Whitney map, but the converse of this assertion is not true. Note that the existence of a strongly admissible Whitney map for $\mathscr{S}$ implies that $F_{1}(X) \cong X$ is a strong deformation retract of $\mathfrak{S}_{\mathcal{E}}$. Hence we have

(2.2) Proposition. Suppose that $X$ is a Peano continuum and $\omega$ is a strongly admissible Whitney map for $\mathfrak{G}=2^{X}$ or $C(X)$. Then $X$ is an AR.

Proof. By [14], $\mathfrak{S}$ is an AR, which implies that $X$ is an AR.

(2.3) TheOREM (cf. [7, (2.14)]). If $X$ is a compact convex subset of a Banach space $B$, then there are strongly admissible Whitney maps for $\mathfrak{S}=2^{X}$ and $C(X)$. 
Proof. Let $\omega$ be the Whitney map for $\mathscr{S}$ as defined in [13, p. 275]. Since $X$ is an $\mathrm{AR}$, there is a retraction $r: \mathfrak{S}_{\mathcal{B}} \rightarrow F_{1}(X) \cong X$. For each $A \in \mathfrak{S}$, define a homotopy $h_{A}: A \times[0,1] \rightarrow X$ by $h_{A}(a, s)=s a+$ $(1-s) r(A)$ for each $a \in A$ and $0 \leq s \leq 1$. Also, define a function $h: \mathfrak{S} \times[0,1] \rightarrow \mathfrak{S}$ by

$$
h(A, s)=\left\{h_{A}(a, s) \mid a \in A\right\} \quad \text { for each } A \in \mathfrak{S} \text { and } 0 \leq s \leq 1 .
$$

It is easily seen that $h$ is continuous. The definition of $h$ implies that $h$ satisfies the conditions (1) and (2) of Definition (2.1). Also, by [7, (2.13)], $h$ satisfies the condition (3) of Definition (2.1). This completes the proof.

(2.4) TheOREM (cf. [7, (2.16)]). If $X$ is any dendrite, then there are strongly admissible Whitney maps for $\mathfrak{S}=2^{X}$ and $C(X)$.

Proof. Let $p \in X$. Let $\leq_{p}, d$ and $D$ be as in [7, (2.16)] and let $\omega$ be the Whitney map for $\mathfrak{S}=2^{X}$ or $C(X)$ as in [13, p. 275]. Since $X$ is an AR, there is a retraction $r: \mathfrak{S} \rightarrow F_{1}(X) \cong X$. For each $A \in \mathfrak{S}$, define a function $h_{A}: A \times[0,1] \rightarrow X$ satisfying the following conditions;

(1) $h_{A}(a, s)$ is contained in the arc from $a$ to $r(A)$, and

(2) if $a \in A$, then $s \cdot D\left(a, h_{A}(a, s)\right)=(1-s) \cdot D\left(h_{A}(a, s), r(A)\right)$.

Then we can easily see that $h_{A}$ is continuous. Also, define a function $h$ : $\mathfrak{S} \times[0,1] \rightarrow \mathfrak{S}$ by

(3) $h(A, s)=\left\{h_{A}(a, s) \mid a \in A\right\}$ for each $A \in \mathscr{S}$ and $0 \leq s \leq 1$.

Then $h$ is continuous. Also, by the definition of $h$ and [7, (2.13)], we can see that $h$ satisfies the conditions (1), (2) and (3) of Definition (2.1). Hence $\omega$ is a strongly admissible Whitney map for $\mathfrak{S}$.

A map $p: E \rightarrow B$ between metric spaces is a strongly regular mapping ([1] and [6]) if it is a proper map and for each $b_{0} \in B$ and $\varepsilon>0$ there is a neighborhood $U\left(b_{0}\right)$ of $b_{0}$ in $B$ such that if $b \in U\left(b_{0}\right)$, there exist maps $g_{b b_{0}}: p^{-1}(b) \rightarrow p^{-1}\left(b_{0}\right)$ and $g_{b_{0} b}: p^{-1}\left(b_{0}\right) \rightarrow p^{-1}(b)$ such that $g_{b b_{0}}, g_{b_{0} b}$ move points no more than $\varepsilon$ and $g_{b b_{0}} g_{b_{0} b}, g_{b_{0} b} g_{b b_{0}}$ are homotopic to the identity maps on $p^{-1}\left(b_{0}\right), p^{-1}(b)$ via homotopies which move points no more than $\varepsilon$, respectively. Note that every strongly regular mapping is an open map.

Then we have the following

(2.5) TheOrem. Let $X$ be a Peano continuum and let $\mathfrak{S}=2^{X}$ or $C(X)$.

(i) If $\omega$ is an admissible Whitney map for $\mathfrak{\mathcal { E }}$, then $\omega \mid \omega^{-1}((0, \omega(X)])$ : $\omega^{-1}((0, \omega(X)]) \rightarrow(0, \omega(X)]$ is a strongly regular mapping with $A R$ fibers. 
(ii) If $\omega$ is a strongly admissible Whitney map for $\mathfrak{S}$, then $\omega: \mathfrak{S} \rightarrow$ $[0, \omega(X)]$ is a strongly regular mapping with AR fibers.

Proof. Suppose that $\omega$ is an admissible Whitney map for $\mathfrak{S}$ and $h$ is an $\omega$-admissible deformation. By [7, (2.9)], $\omega^{-1}(t)$ is a compact AR for each $0<t \leq \omega(X)$. Let $t \in(0, \omega(X))$. Define $\theta_{t}: \omega^{-1}([t, \omega(X)]) \rightarrow[0,1]$ by

$$
\theta_{t}(A) \equiv \text { the unique number in }[0,1] \text { such that } \omega\left(h\left(A, \theta_{t}(A)\right)\right)=t
$$

Define $r_{t}: \omega^{-1}([t, \omega(X)]) \rightarrow \omega^{-1}(t)$ by

$$
r_{t}(A)=h\left(A, \theta_{t}(A)\right) \quad(\text { see }[7,(2.7)]) .
$$

Now let $t \in(0, \omega(X)]$. Let $K_{d}$ be as in [7, (1.2)]. Define $\Psi_{t}: \omega^{-1}([0, t]) \rightarrow$ $[0, \infty)$ by

$$
\begin{aligned}
& \Psi_{t}(A) \equiv \text { the smallest number in }[0, \infty) \text { such that } \\
& \qquad \omega\left(K_{d}\left(A, \Psi_{t}(A)\right)\right)=t .
\end{aligned}
$$

Define $R_{t}: \omega^{-1}([0, t]) \rightarrow \omega^{-1}(t)$ by

$$
R_{t}(A)=K_{d}\left(A, \Psi_{t}(A)\right) .
$$

Now suppose that $0<t_{1}<t_{2} \leq \omega(X)$. Define mappings $g_{t_{1} t_{2}}: \omega^{-1}\left(t_{1}\right) \rightarrow$ $\omega^{-1}\left(t_{2}\right)$ and $g_{t_{2} t_{1}}: \omega^{-1}\left(t_{2}\right) \rightarrow \omega^{-1}\left(t_{1}\right)$ by

$$
g_{t_{1} t_{2}}=R_{t_{2}} \mid \omega^{-1}\left(t_{1}\right) \text { and } g_{t_{2} t_{1}}=r_{t_{1}} \mid \omega^{-1}\left(t_{2}\right) \text {. }
$$

To show that $g_{t_{2} t_{1}} \circ g_{t_{1} t_{2}}$ is homotopic to the identity map on $\omega^{-1}\left(t_{1}\right)$, define $H_{1}: \omega^{-1}\left(t_{1}\right) \times[0,1] \rightarrow \omega^{-1}\left(t_{1}\right)$ by

$$
H_{1}(A, s)=r_{t_{1}}\left(K_{d}\left(A, s \cdot \Psi_{t_{2}}(A)\right)\right) .
$$

To show that $g_{t_{1} t_{2}} \circ g_{t_{2} t_{1}}$ is homotopic to the identity map on $\omega^{-1}\left(t_{2}\right)$, define $H_{2}: \omega^{-1}\left(t_{2}\right) \times[0,1] \rightarrow \omega^{-1}\left(t_{2}\right)$ by

$$
H_{2}(A, s)=R_{t_{2}}\left(h\left(A, 1-s\left(1-\theta_{t_{1}}(A)\right)\right)\right) .
$$

By using maps $g_{t_{1} t_{2}}, g_{t_{2} t_{1}}$ and homotopies $H_{1}, H_{2}$, we can easily see that $\omega \mid \omega^{-1}((0, \omega(X)]): \omega^{-1}((0, \omega(X)]) \rightarrow(0, \omega(X)]$ is a strongly regular mapping. Finally, if $\omega$ is a strongly admissible Whitney map for $\mathfrak{S}$, the case when $t_{1}=0$ can be handled in the analogous fashion. This completes the proof. 
3. Trivial bundle maps and Whitney maps. A map $p: E \rightarrow B$ is a trivial bundle map with $F$ fibers if there is a homeomorphism $h: E \stackrel{\approx}{\rightarrow} B \times F$ such that the following diagram is commutative;

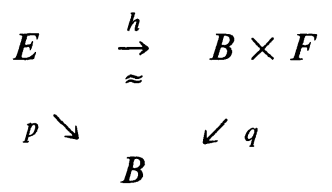

where $q: B \times F \rightarrow B$ is the projection map, i.e., $q(b, f)=b$ for $(b, f) \in$ $B \times F$.

Then we have the following

(3.1) Theorem. Let $X$ be a Peano continuum and let $\mathfrak{S}=2^{X}$ or $C(X)$. If $\mathfrak{S}=C(X)$, assume that $X$ contains no free arc.

(i) If $\omega$ is an admissible Whitney map for $\mathfrak{S}$, then $\omega \mid \omega^{-1}((0, \omega(X)))$ : $\omega^{-1}((0, \omega(X))) \rightarrow(0, \omega(X))$ is a trivial bundle map with Hilbert cube fibers.

(ii) If $X$ is the Hilbert cube $Q$, then there is a strongly admissible Whitney map for $\mathfrak{S}$ such that $\omega \mid \omega^{-1}([0, \omega(X))): \omega^{-1}([0, \omega(X))) \rightarrow$ $[0, \omega(X))$ is a trivial bundle map with Hilbert cube fibers.

To prove (3.1) we need the following result of Chapman and Ferry.

(3.2) THEOREM ([3]). If $p: E \rightarrow B$ is a strongly regular mapping with compact $Q$-manifold fibers and $\operatorname{dim} B<\infty$, then $p$ is a locally trivial bundle map.

Proof of (3.1). Case (i). It follows from (2.5) that $\omega \mid \omega^{-1}((0, \omega(X)))$ : $\omega^{-1}((0, \omega(X))) \rightarrow(0, \omega(X))$ is a strongly regular mapping. By $(1.1), \omega^{-1}(t)$ is a Hilbert cube for each $0<t<\omega(X)$. Note that $\operatorname{dim}(0, \omega(X))=1$. Hence by (3.2), $\omega \mid \omega^{-1}((0, \omega(X)))$ is a locally trivial bundle map with Hilbert cube fibers. Since $(0, \omega(X))$ is contractible, $\omega \mid \omega^{-1}((0, \omega(X)))$ is a trivial bundle map.

Case (ii). We may assume that $Q$ is a compact convex subset of the Hilbert space $l_{2}$. By (2.3), there is a strongly admissible Whitney map $\omega$ for $\mathfrak{S} .(1.1),(2.5)$ and (3.2) imply that $\omega \mid \omega^{-1}([0, \omega(X))): \omega^{-1}([0, \omega(X)))$ $\rightarrow[0, \omega(X))$ is a trivial bundle map with Hilbert cube fibers. This completes the proof.

Note that every trivial bundle map is a strongly regular mapping. In the statement (i) of (2.5) (resp., (3.1)), we can not conclude that $\omega \mid \omega^{-1}([0, \omega(X)))$ is a strongly regular mapping (resp., a trivial bundle 
map). In fact, we have the following

(3.3) Proposition. Let $X$ be a Peano continuum and let $\omega$ be an admissible Whitney map for $\mathfrak{S}=2^{X}$ or $C(X)$. If $\omega \mid \omega^{-1}([0, \omega(X)))$ : $\omega^{-1}([0, \omega(X))) \rightarrow[0, \omega(X))$ is a strongly regular mapping, then $X$ is an $A R$. In particular, if $X$ is the topological cone over a locally connected compactum $Y$ which is not an ANR, then each admissible Whitney map for $\mathfrak{S}$ is not a strongly regular mapping.

Proof. By [7, (2.9)], $\omega^{-1}(t)$ is a compact AR for each $0<t \leq \omega(X)$. If $\omega \mid \omega^{-1}([0, \omega(X)))$ is a strongly regular mapping, for any $\varepsilon>0$ there is a positive Whitney level $\omega^{-1}(t)(t>0)$ such that $X$ is $\varepsilon$-homotopically dominated by $\omega^{-1}(t)$. By Dowker's result [Homotopy extension theorems, Proc. London Math. Soc. 6 (1959), p. 105], $X$ is an ANR. Since $X$ is contractible, $X$ is an AR.

Naturally, the following question is raised.

(3.4) Question. If $X$ is a compact $\mathrm{AR}$, is there a strongly admissible Whitney map for $2^{X}$ or $C(X)$ ?

Finally, we shall prove the following

(3.5) THEOREM. Let $X$ be the $n$-sphere $S^{n}(n=1,2, \ldots$,$) . Then there is$ a Whitney map $\omega$ for $\mathfrak{S}=2^{S^{n}}(n=1,2, \ldots$,$) or C\left(S^{n}\right)(n=2,3, \ldots$,$) such$ that for some $t_{0} \in(0, \omega(X)), \omega \mid \omega^{-1}\left(\left[0, t_{0}\right]\right): \omega^{-1}\left(\left[0, t_{0}\right]\right) \rightarrow\left[0, t_{0}\right]$ is a strongly regular mapping. Moreover, $\omega \mid \omega^{-1}\left(\left(0, t_{0}\right]\right): \omega^{-1}\left(\left(0, t_{0}\right]\right) \rightarrow\left(0, t_{0}\right]$ is a trivial bundle map with $X \times Q$ fibers.

To prove (3.5), we need Torunczyk's characterization of compact $Q$-manifolds.

(3.6) TheOrem ([13, Theorem 1]). Let $X$ be a compact ANR. If the identify map on $X$ is a uniform limit of Z-maps, then $X$ is a compact $Q$-manifold (for the definition of $Z$-map, see [13]).

Proof of (3.5). Assume that $X=S^{n}=\left\{x=\left(x_{1}, x_{2}, \ldots, x_{n+1}\right) \in\right.$ $\left.R^{n+1} \mid\|x\|=1\right\} \subset R^{n+1}$. Let $d$ be the metric on $S^{n}$ such that for each $x=\left(x_{1}, x_{2}, \ldots, x_{n+1}\right)$ and $y=\left(y_{1}, y_{2}, \ldots, y_{n+1}\right) \in S^{n}$,

$$
d(x, y)=\arccos \left[x_{1} y_{1}+x_{2} y_{2}+\cdots+x_{n} y_{n}+x_{n+1} y_{n+1}\right] \text {. }
$$


Note that if $d(x, y)<\pi$ and $0 \leq s \leq 1$, there is only one point $h_{s}(x, y)$ $\in S^{n}$ such that

(1) $d\left(x, h_{s}(x, y)\right)+d\left(h_{s}(x, y), y\right)=d(x, y)$, and

(2) $d\left(x, h_{s}(x, y)\right)=s \cdot d(x, y)$ (e.g., see [2, p. 327]).

Since $F_{1}(X) \cong X$ is an ANR, there is a neighborhood $U$ of $F_{1}(X)$ in $\mathfrak{g}$ and a retraction $r: U \rightarrow F_{1}(X)$. Choose a positive number $t_{1} \in(0, \omega(X))$ such that

(3) $\omega^{-1}\left(\left[0, t_{1}\right]\right) \subset U$ and

(4) $d(r(A), a)<\pi / 2$ for each $A \in \omega^{-1}\left(\left[0, t_{1}\right]\right)$ and $a \in A$.

Define a function $h_{A}: A \times[0,1] \rightarrow X$ by

(5) $h_{A}(a, s)=h_{s}(r(A), a)$ for each $a \in A$ and $0 \leq s \leq 1$.

Then $h_{A}$ is continuous. Also, define a function $h: \omega^{-1}\left(\left[0, t_{1}\right]\right) \times[0,1] \rightarrow \mathfrak{S}$ by

(6) $h(A, s)=\left\{h_{A}(a, s) \mid a \in A\right\}$ for each $A \in \omega^{-1}\left(\left[0, t_{1}\right]\right), 0 \leq s \leq 1$. Then $h$ is continuous and the definition of $h$ and [7, (2.13)] imply that $h$ satisfies the following conditions.

(7) $h(\{x\}, s)=\{x\}$ for each $\{x\} \in F_{1}(X), 0 \leq s \leq 1$,

(8) $h(A, 1)=A, h(A, 0)=\{r(A)\} \in F_{1}(X)$ for each $A \in \omega^{-1}\left(\left[0, t_{1}\right]\right)$ and

(9) if $A \in \omega^{-1}\left(\left[0, t_{1}\right]\right)$ and $\omega(h(A, s))>0$ for some $0<s \leq 1$, then $\omega(h(A, s))>\omega\left(h\left(A, s^{\prime}\right)\right)$ for each $0 \leq s^{\prime}<s \leq 1$.

Choose a positive number $t_{0}\left(<t_{1}\right)$. By (9), we have

(10) $h\left(\omega^{-1}\left(\left[0, t_{0}\right]\right) \times[0,1]\right) \subset \omega^{-1}\left(\left[0, t_{0}\right]\right)$.

also, by using the homotopy $K_{d}$ (see [7, (1.2)]) and by the same way as in [7, (2.7)], $\omega^{-1}(t)$ is a retract of $\omega^{-1}\left(\left[0, t_{1}\right)\right)$ for each $0 \leq t \leq t_{0}$. Since $\omega^{-1}\left(\left[0, t_{1}\right)\right)$ is an ANR, $\omega^{-1}(t)$ is also an ANR. By using (3.6) and the same argument as in $[7, \S 3,4]$, we can see that $\omega^{-1}(t)$ is a compact $Q$-manifold for each $0<t \leq t_{0}$. Also, by using the homotopy $h$, we can see that $\omega \mid \omega^{-1}\left(\left[0, t_{0}\right]\right): \omega^{-1}\left(\left[0, t_{0}\right]\right) \rightarrow\left[0, t_{0}\right]$ is a strongly regular mapping (see (2.5)). Hence by $(3.2), \omega \mid \omega^{-1}\left(\left(0, t_{0}\right]\right)$ is a trivial bundle map with compact $Q$-manifold fibers. Let $\omega^{-1}(t)=M$ for some $t \in\left(0, t_{0}\right)$. Consider the map $g=q \circ\left(\left(\omega \mid \omega^{-1}\left(\left[0, t_{0}\right]\right)\right) \times 1_{q}\right): \omega^{-1}\left(\left[0, t_{0}\right]\right) \times Q \rightarrow\left[0, t_{0}\right] \times$ $Q \rightarrow\left[0, t_{0}\right]$, where $1_{Q}: Q \rightarrow Q$ denotes the identity map on $Q$ and $q$ : $\left[0, t_{0}\right] \times Q \rightarrow\left[0, t_{0}\right]$ is the projection map. Then $g$ is also a strongly regular mapping with compact $Q$-manifold fibers, hence $g$ is a trivial bundle map with $M \times Q$ fibers. In particular, $M \cong M \times Q \cong g^{-1}(0) \cong$ $S^{n} \times Q$. This completes the proof.

(3.7) REMARK. In the proof of (3.5), we cannot conclude that $\omega \mid \omega^{-1}((0, \omega(X)))$ is a trivial bundle map with $S^{n} \times Q$ fibers. In [7, (4.15)], Goodykoontz and Nadler showed the following fact: Let $X=S^{1}$ be the 
unit circle and let $\omega$ be the Whitney map for $2^{X}$ as defined in the proof (3.5). Then there is a positive number $t_{2}, t_{2}<\omega(X)$, such that $\omega^{-1}\left(t_{2}\right)$ has a finite-dimensional nonempty open subset of $\omega^{-1}\left(t_{2}\right)$. Hence $\omega^{-1}\left(t_{2}\right)$ is not a $Q$-manifold.

(3.8) COROllaRY. There is a Whitney map $\omega$ for $\mathfrak{Y}=2^{S^{n} \times Q}$ or $C\left(S^{n} \times Q\right), \quad(n=1,2, \ldots)$ such that for some $t_{0} \in\left(0, \omega\left(S^{n} \times Q\right)\right)$, $\omega \mid \omega^{-1}\left(\left[0, t_{0}\right]\right)$ is a trivial bundle map with $S^{n} \times Q$ fibers.

\section{REFERENCES}

[1] D. A. Addis, A strong regularity condition of mappings, General Topology and Appl., 2 (1972), 199-213.

[2] K. Borsuk, On a metrization of polytopes, Fund. Math., 47 (1959), 325-341.

[3] T. A. Chapman and S. Ferry, Hurewicz fiber maps with ANR fibers, Topology, 16 (1977), 131-144.

[4] D. W.Curtis and R. M. Schori, Hyperspaces of Peano continua are Hilbert cubes, Fund. Math., 101 (1978), 19-36.

[5] _ Hyperspaces which characterize simple homotopy type, General Topology and Appl., 6 (1976), 153-165.

[6] S. Ferry, Strongly regular mappings with compact ANR fibers are Hurewicz fiberings, Pacific J. Math., 75 (1978), 373-382.

[7] J. T. Goodykoontz, Jr. and S. B. Nadler, Jr., Whitney levels in hyperspaces of certain Peano continua, Trans. Amer. Math. Soc., 274 (1982), 671-694.

[8] J. L. Kelley, Hyperspaces of a continuum, Trans. Amer. Math. Soc., 52 (1942), 22-36.

[9] S. B. Nadler Jr., A characterization of locally connected continua by hyperspace retractions, Proc. Amer. Math. Soc., 67 (1977), 167-176.

[10] Hyperspaces of sets, Pure and Appl. Math., 49 Dekker, New York, 1978.

[11] A. Petrus, Contractibility of Whitney continua in $C(X)$, General Topology and Appl., 9 (1978), 275-288.

[12] H. Torunczyk, On CE-images of Hilbert cube and characterization of Q-manifolds, Fund. Math., 106 (1980), 31-40.

[13] H. Whitney, Regular families of curves I, Proc. Nat. Acad. Sci. U.S.A., 18 (1932), 275-278.

[14] M. Wojdyslawski, Sur la contractilité des hyeprspaces des continus localement connexes, Fund. Math., 30 (1938), 247-252.

Received April 29, 1983 and in revised form January 17, 1984.

UNIVERSITY OF TSUKUBA

IBARAKI 305, JAPAN 



\section{PACIFIC JOURNAL OF MATHEMATICS EDITORS}

\author{
V. S. VARADARAJAN (Managing Editor) \\ University of California \\ Los Angeles, CA 90024 \\ Charles R. DePrima \\ California Institute of Technology \\ Pasadena, CA 91125 \\ R. FINN \\ Stanford University \\ Stanford, CA 94305
}

\author{
HeRmanN FlaschKa \\ University of Arizona \\ Tucson, AZ 85721 \\ Ramesh A. Gangolli \\ University of Washington \\ Seattle, WA 98195 \\ ROBION KIRBY \\ University of California \\ Berkeley, CA 94720
}

C. C. MOORE

University of California

Berkeley, CA 94720

H. SAMELSON

Stanford University

Stanford, CA 94305

HAROLD STARK

University of California, San Diego

La Jolla, CA 92093

ASSOCIATE EDITORS
R. ARENS
E. F. BECKENBACH
B. H. NeUmanN
F. WOLF
K. YosHIDA (1906-1982)

\section{SUPPORTING INSTITUTIONS}

UNIVERSITY OF ARIZONA

UNIVERSITY OF BRITISH COLUMBIA

CALIFORNIA INSTITUTE OF TECHNOLOGY

UNIVERSITY OF CALIFORNIA

MONTANA STATE UNIVERSITY

UNIVERSITY OF NEVADA, RENO

NEW MEXICO STATE UNIVERSITY

OREGON STATE UNIVERSITY
UNIVERSITY OF OREGON

UNIVERSITY OF SOUTHERN CALIFORNIA

STANFORD UNIVERSITY

UNIVERSITY OF HAWAII

UNIVERSITY OF TOKYO

UNIVERSITY OF UTAH

WASHINGTON STATE UNIVERSITY

UNIVERSITY OF WASHINGTON 


\section{Pacific Journal of Mathematics}

Vol. 119, No. $1 \quad$ May, 1985

Maurice Chacron, Nonisotropic unitary spaces and modules with

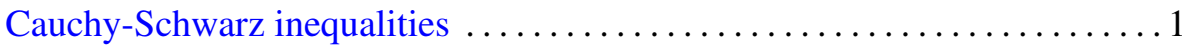

Myriam Dechamps-Gondim, Françoise Piquard and H. Queffélec, On

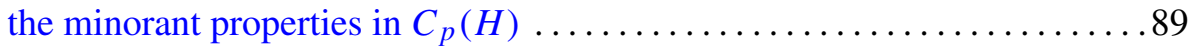

Klaus Floret and V. B. Moscatelli, On bases in strict inductive and projective limits of locally convex spaces .................... 103

Norman Joseph Goldstein, Degenerate secant varieties and a problem on

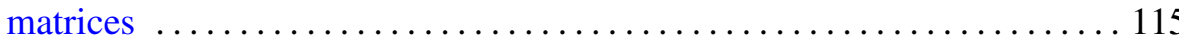

Harold Morris Hastings and Stefan Waner, $G$-bordism with singularities

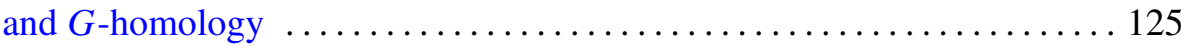

Takesi Isiwata, Clopen realcompactification of a mapping ........... 153

Hisao Kato, Concerning hyperspaces of certain Peano continua and strong

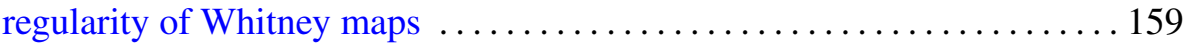

Elyahu Katz and Sidney Allen Morris, Free products of topological groups

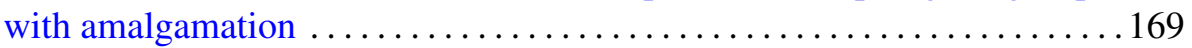

Kyewon Koh Park, Nice dense subsets for ergodic flows and Bernoulli

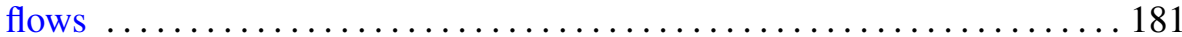

Francis Pastijn and Peter George Trotter, Lattices of completely regular

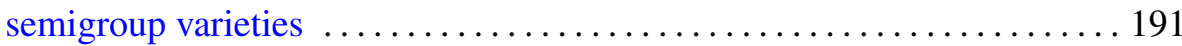

Rae Michael Andrew Shortt, Reticulated sets and the isomorphism of analytic powers

David A. Stegenga and Kenneth R. Stephenson, Generic covering properties for spaces of analytic functions

M. V. Subba Rao and R. Sitaramachandra Rao, On some infinite series of

L. J. Mordell and their analogues 